\title{
Lidar-based wake tracking for closed-loop wind farm control
}

\author{
Steffen Raach, David Schlipf, and Po Wen Cheng \\ Stuttgart Wind Energy (SWE), University of Stuttgart, Allmandring 5B, 70569 Stuttgart, Germany \\ Correspondence to: Steffen Raach (raach@ifb.uni-stuttgart.de)
}

Received: 10 January 2017 - Discussion started: 24 January 2017

Revised: 22 March 2017 - Accepted: 14 April 2017 - Published: 23 May 2017

\begin{abstract}
This work presents two advancements towards closed-loop wake redirection of a wind turbine. First, a model-based wake-tracking approach is presented, which uses a nacelle-based lidar system facing downwind to obtain information about the wake. The method uses a reduced-order wake model to track the wake. The wake tracking is demonstrated with lidar measurement data from an offshore campaign and with simulated lidar data from a simulation with the Simulator fOr Wind Farm Applications (SOWFA). Second, a controller for closedloop wake steering is presented. It uses the wake-tracking information to set the yaw actuator of the wind turbine to redirect the wake to a desired position. Altogether, the two approaches enable a closed-loop wake redirection.
\end{abstract}

\section{Introduction}

In recent years, wind farm control has gained more and more importance in the wind energy control community, due to interactions between individual wind turbines in a wind farm. The wind speed in the wake of a wind turbine is reduced with respect to the free-stream wind speed. Additionally, the turbulence in the wake is increased. If a wind turbine is impacted by a wake from a wind turbine located upwind, the wind turbine produces less power and is faced with higher structural loads because of the increased turbulence; see Borisade et al. (2015). Describing the wake effects and quantifying the decay has been of interest in research for years. Different models have been developed to address different wake properties, such as the velocity deficit and the increased turbulence intensity. There are empirical models, data-driven models, and models which describe the physical behavior in the wake, all varying in complexity and computational effort. Mainly, models with low complexity are steadystate models which describe the interaction in a static manner and no wake propagation is modeled. Further research is needed to develop control-oriented dynamic wake models.

The same two goals are valid for both wind turbine and wind farm control: (1) maximization of the total power and (2) reduction of the structural loads. Two main concepts have been introduced for wind farm control: (1) axial induction control and (2) wake redirection control. Axial induction control aims at manipulating the axial induction by the blade pitch or torque actuator and operating the wind turbine at a lower production level. This results in a lower wake deficit and aims at minimizing structural load effects on the downwind wind turbines and preserving energy in the flow. The effect on the overall energy capture of the wind farm is not clear yet. See Boersma et al. (2017) for a general overview on wind farm control.

The idea of redirecting the wake by the yaw actuator instead of trying to mitigate its intensity has been discussed in different publications; see Fleming et al. (2014a, b) and Gebraad et al. (2014). In simulation studies it was shown that the wake is redirected up to 0.54 times the rotor diameter, $D$, (at a seven $D$ downwind distance) by yawing the turbine up to $40^{\circ}$; see Fleming et al. (2014b). Different investigations have shown promising results when improving the power output of a wind farm by applying yaw offsets in open-loop approaches; see Gebraad et al. (2014) and Fleming et al. (2014a). Nevertheless, the form in which it has been applied so far contains drawbacks: (1) applying optimized yaw angles in a feed-forward approach does not guarantee that the wake is going in the desired direction and thus, the quality of the model, which is used to compute the yaw angles, highly influences the control performance. (2) There is 
no observation of whether the wake is being redirected correctly. The concept of closed-loop wake redirection, which was introduced in Raach et al. (2016) can help to overcome the drawbacks.

A major barrier for wind farm control applications has been the lack of measurement devices with which to measure the flow interactions between wind turbines, but also the cost and availability of the devices. Further, modeling the three dimensional flow field is not a straightforward approach, since the flow is usually described by the Navier-Stokes equations. Lidar can be a useful tool for addressing the measurement problem in wind farm applications while bearing in mind the instrument limitations and the assumptions required to extract the information and exploit the lidar measurement data.

This paper addresses the wind farm control concept of wake redirection. It aims to enable closed-loop wake redirection by presenting a method to obtain the wake position using lidar measurements. Further, the difficulty in wake position definition and measurability is discussed. A model-based estimation approach is presented and used to obtain important quantities for wake redirection using a nacelle-based lidar system facing downwind, and a closed loop controller is designed and analyzed. In summary, this work presents an entire concept for lidar-based closed-loop wake redirection.

\section{Methodology}

In order to enable lidar-based closed-loop wake redirection within a wind farm, two main tasks must be considered: (1) the measurement task and (2) the control task. This work focuses mainly on the measurement task but also gives a summary of a solution to the control task, which was presented in Raach et al. (2016). Figure 1 presents the general concept of closed-loop wake redirection and the link between the measurement and control task.

\subsection{Problem formulation for wake tracking}

The main issue in the context of wake-tracking algorithms is that no clear definition exists for the wake center. Moreover, the idea of a wake center is based on time-averaged profiles of the wake behind a turbine (1 to $10 \mathrm{~min}$ averages). Averaging the flow yields a (double) Gaussian function for the velocity deficit profile in the horizontal and vertical directions. From this a wake center can then be defined. However, when different methods are used to define the shape, wake center estimates may vary under the same flow conditions; see Vollmer et al. (2016). The absence of a unique wake center definition must be considered when comparing results. Furthermore, this means that, even with full-flow field information, the wake center is not a measurable quantity and depends on the definition. See also Doubrawa et al. (2017) and Howland et al. (2016) for a review of wake center estimation methods. The task of lidar-based wake tracking first includes a reference definition of the wake center. Then, the result of

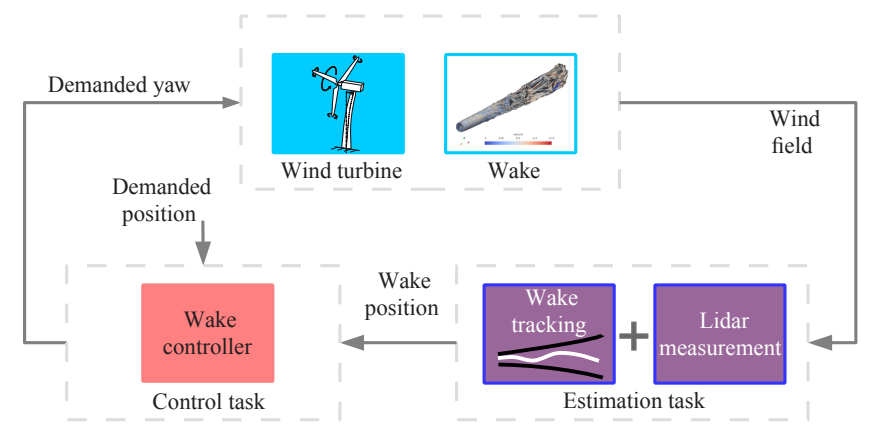

Figure 1. The conceptual diagram of closed-loop wake redirection and its two main tasks: (1) the estimation task addressed in Sect. 5 and (2) the control task addressed in Sect. 6.

the estimation method from the lidar measurement data can be compared to the reference definition.

\subsection{The estimation task}

Measuring flow quantities is crucial for enabling a closedloop controller to influence the wake properties. The task of the measurement problem is to provide the necessary quantities for the controller. This means using a measurement device, such as a lidar, and processing the measurement data in such a way that they are useful for the controller. Since the lidar measurement principle has several limitations in providing wind field information, an adequate estimation technique is used as described in Sect. 5.

\subsection{The control task}

The second task towards a closed-loop wake redirection is the control task. Its main challenge is to convert the estimated wake position information and its desired value to a demanded yaw signal. A feedback controller has to be designed which steers the wake center to the desired position and compensates for uncertainties in the models. Since the reaction of a change in yaw is measured with a delay due to the wake propagation time, the controller has to be designed to overcome this limitation.

In the following section, the measurement problem is addressed first. A method is presented to estimate wake information from lidar measurement data using a nacelle-based lidar system. Second, the controller problem is addressed in Sect. 6. A wake redirection controller is presented which uses the obtained wake information, (namely the wake center position), and steers the wake using the yaw actuator. The overall goal of this paper is to present the framework of lidarbased closed-loop wake redirection while providing example models and controller. 


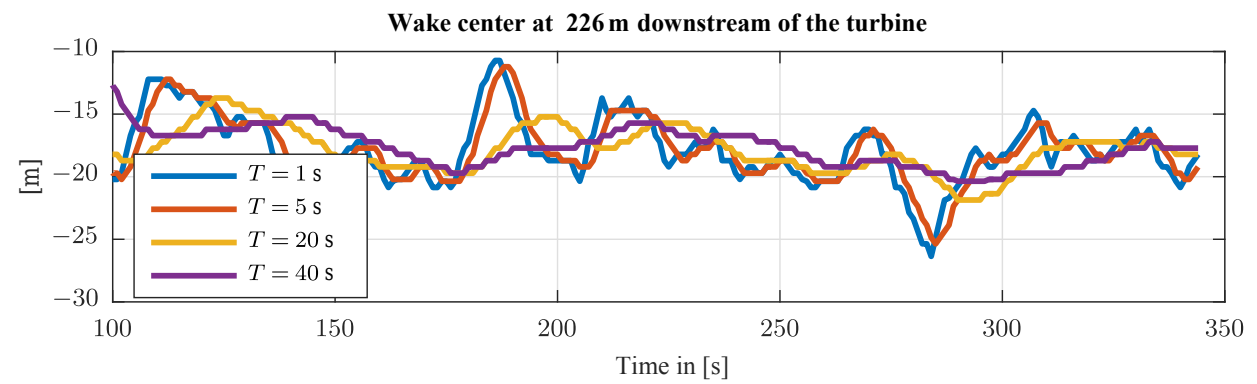

Figure 2. Time evolution of wake center (at a $1.8 \mathrm{D}$ downwind distance) when different window lengths $T$ are used to average the flow during the wake center calculation.

\section{Reference definition and its impact on the estimation task}

In this section the wake center definition is addressed through the use of simulation data, which can cover a larger area at a higher spatial and temporal resolution than measurements would be able to provide.

\subsection{Wake center definition}

As previously mentioned, it is first necessary to define the wake center. The minimum wind power method proposed by Vollmer et al. (2016) is adopted and modified to identify the wake center. Thus, it is defined as the downstream position at which a hypothetical turbine of identical characteristics and yaw angle would produce the least power. This yields the minimization problem

$\min _{y} \int_{0}^{2 \pi} \int_{y}^{R+y} u(r, \phi)^{3} r \mathrm{~d} r \mathrm{~d} \phi$,

where the position of the turbine is at $y$ (lateral offset) and $z=0$ (hub-height) and the rotor area is described in the polar coordinate system $(r, \phi)$. The definition then assumes that the wake center is at $(y, z)$.

The flow field is time averaged over different running window lengths and the impact of the window lengths is analyzed. The calculated wake center (at a $1.8 D$ downwind distance) obtained with a running averaged filter with different window lengths is presented in Fig. 2. The presented results are for a low turbulence intensity $(\mathrm{TI}=6 \%)$ SOWFA simulation under a mean hub-height free-stream wind speed $8 \mathrm{~m} \mathrm{~s}^{-1}$. The available flow field data has a sampling frequency of $1 \mathrm{~Hz}$ and the wake center is calculated from each sample. The wake center clearly converges to a steady value with increasing averaging time $T$. An increased averaging time, however, slows the adjustment, e.g., to a changing wind direction or a set point change, and should be considered when choosing an averaging time.

\subsection{Problem discussion of lidar-based wake tracking}

The problem of wake center estimation is different from other problems in lidar-based wind field reconstruction. Other model-based approaches in wind field reconstruction (e.g., estimation of the rotor-effective wind speed, or of $u$ and $v$ wind vector components using lidar measurements as in Schlipf et al., 2012) can first be compared to existing quantities. Further, the models can be used to predict line-of-sight velocities $\left(v_{\text {los }}\right)$ of lidar measurements and be directly compared to the real data. Therefore, the model can be used in two directions, estimating and predicting the wind field.

When the wake center is defined as in Eq. (1) the prediction of the wind field from a given position is not possible and neither is a direct comparison of line-of-sight data. Nevertheless, the wake center position definition is used as a reference because of its robustness and simplicity.

\section{A simplified wake model for wake tracking}

The estimation task addresses the processing and estimation of useful information and provides them to the controller. Since a lidar system has several limitations, the desired quantities, like the wake position or the wake deficit, are not directly measurable and have to be estimated from the measurement data. One main limitation of wind lidar systems is that they return the projection of the wind speeds along the direction of the laser beam. This means that a lidar system only provides scalar information of the actual wind vectors. Further, the wind speed is not measured at a certain point but in a volume around the desired measurement location. A solution to these limitations is to implement model-based wind field reconstruction. Wind field reconstruction methods have been developed and used for different applications of lidar systems in wind energy, for example static two- and threedimensional (Schlipf et al., 2012) and dynamic three dimensional wind field reconstruction methods (Raach et al., 2014), approaches for floating lidar systems, Schlipf et al. (2012), as well as for wake measurements Lundquist et al. (2015). Here, the concept of wind field reconstruction is used to obtain information about the wake. 


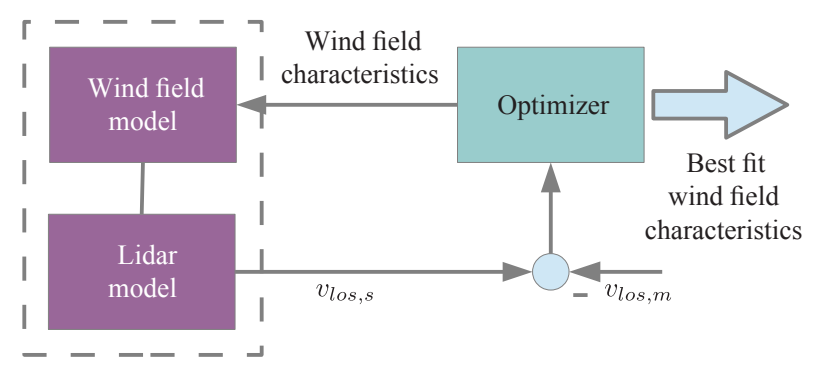

Figure 3. The general concept of model-based wind field reconstruction, in which the wind field characteristics are estimated by fitting simulated lidar measurement data $\left(v_{\mathrm{los}, \mathrm{s}}\right)$ to measurements $\left(v_{\mathrm{los}, \mathrm{m}}\right)$.

The general approach of wind field reconstruction from lidar data is to estimate wind field characteristics from an internal model by fitting simulated lidar data to the measured ones. In Fig. 3 the basic idea of model-based wind field reconstruction is shown. An optimizer is used to find the best fit for a model of the assumed wind field with the defined lidar configuration. The optimizer minimizes the square error of the modeled (simulated) $v_{\mathrm{los}, \mathrm{s}}$ and the measured $v_{\mathrm{los}, \mathrm{m}}$ lidar line-of-sight velocities and returns the estimated model parameter (e.g., wake center position, wake decay, wake deficit, etc.).

In this work, a lidar and a wind field model is used. The wind field model consists of a background wind field model, which defines the ambient wind speed and its profile, and a wake model. The wake model includes the main wake effects: wake deficit, wake evolution, and wake center displacement. The models are presented in the following section.

\subsection{Wind field model}

Figure 4 shows the subparts of the wind field model: (1) the underlaying wind field and (2) the wake model.

The wind field model is described in a wind coordinate system which is denoted by the subscript W. It is rotated horizontally with respect to the global inertial coordinate system I and aligned with the wind direction. The wind speed vector in the W-system is transformed in the I-system by

$$
\left[\begin{array}{c}
u \\
v \\
w
\end{array}\right]_{\mathrm{I}}=\left[\begin{array}{ccc}
\cos \alpha & -\sin \alpha & 0 \\
\sin \alpha & \cos \alpha & 0 \\
0 & 0 & 1
\end{array}\right]\left[\begin{array}{c}
u \\
v \\
w
\end{array}\right]_{\mathrm{W}},
$$

where $\alpha$ is the horizontal rotation of the wind field. The underlying wind field includes the rotor effective wind speed $v_{0}$ and vertical linear shear $\delta_{V}$. It is assumed that the wind field has only a $u$ component. Thus, in the $\mathrm{W}$ coordinate system, the underlying wind field vector at point $i$ with the coordinates $\left[x_{i}, y_{i}, z_{i}\right]^{\mathrm{T}}$ is

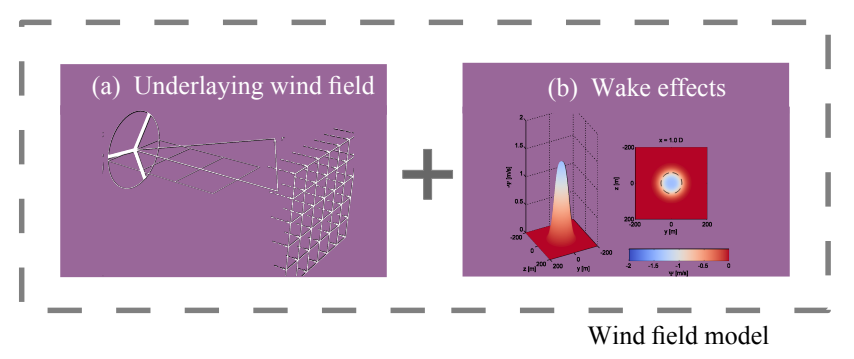

Figure 4. The submodels of the wind field model (in the wind coordinate system $W$ ): (1) the underlaying wind field and (2) the wake model.

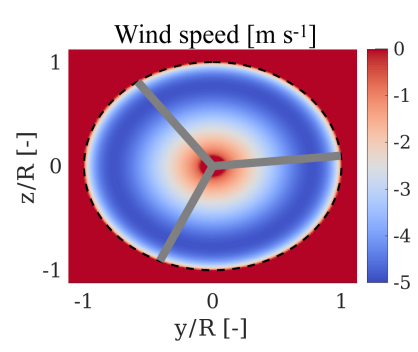

Figure 5. The initial wake deficit directly evaluated at the rotor (at $0 \mathrm{~m}$ downstream). The mean hub-height wind speed $\left(8 \mathrm{~m} \mathrm{~s}^{-1}\right)$ was removed for simplicity. No yaw misalignment is applied.

$\left[\begin{array}{c}u \\ v \\ w\end{array}\right]_{i, \mathrm{~W}}=\left[\begin{array}{c}v_{0}+z_{i} \delta_{V} \\ 0 \\ 0\end{array}\right]$,

where $z_{i}$ is the height above the ground. This is illustrated in Fig. 4 on the left. Further, the wind field is linearly overlaid with the wake model $\Psi$ for the $u$ and $v$ components yielding

$\left[\begin{array}{c}u \\ v \\ w\end{array}\right]_{i, \mathrm{~W}}=\left[\begin{array}{c}v_{0}+z_{i} \delta_{V}+\Psi_{u, i} \\ \Psi_{v, i} \\ 0\end{array}\right]$.

In the following section, the considered wake effects are described and the wake model is presented.

\subsubsection{Wake deficit and wake evolution model}

The wake deficit is modeled with an initial wake deficit at the rotor disk with tip and root losses depending on the energy extraction. In order to get the initial deficit, the energy extraction is mapped by applying Prandtl's root and tip loss function $\Gamma_{\text {Prandtl }}$. Applying the energy conservation assumption yields

$\left(v_{0}+s \Gamma_{\text {Prandtl }}\right)^{2}-\left(1-c_{\mathrm{P}}\right) v_{0}=0$,

with the power coefficient $c_{\mathrm{P}}$. Solving this equation for $s$ gives the initial wake deficit

$\Psi_{\text {init }}=s_{\text {solution }} \Gamma_{\text {Prandtl }}$. 

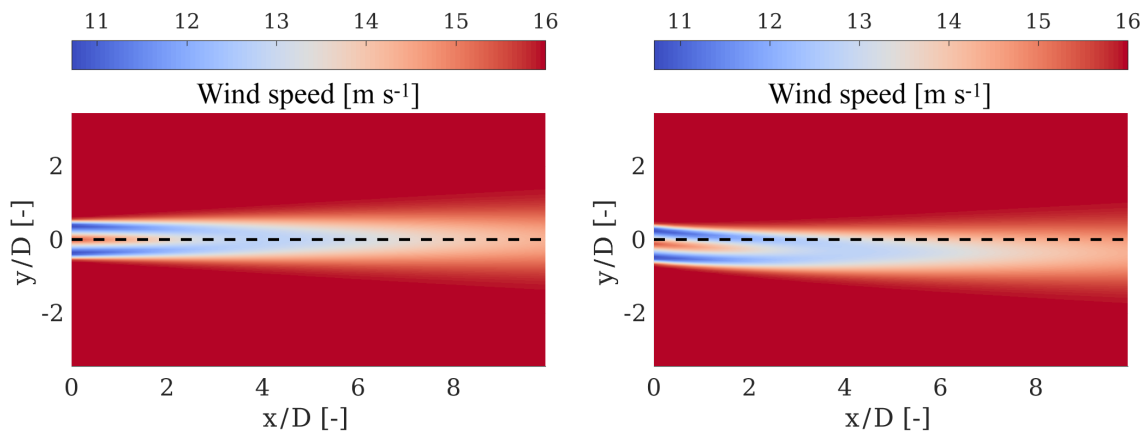

Figure 6. Visualization of two wake situations within a constant wind field of $v_{0}=16 \mathrm{~m} \mathrm{~s}^{-1}$, axial induction $a=0.15$, and dissipation rate $\epsilon=0.1$
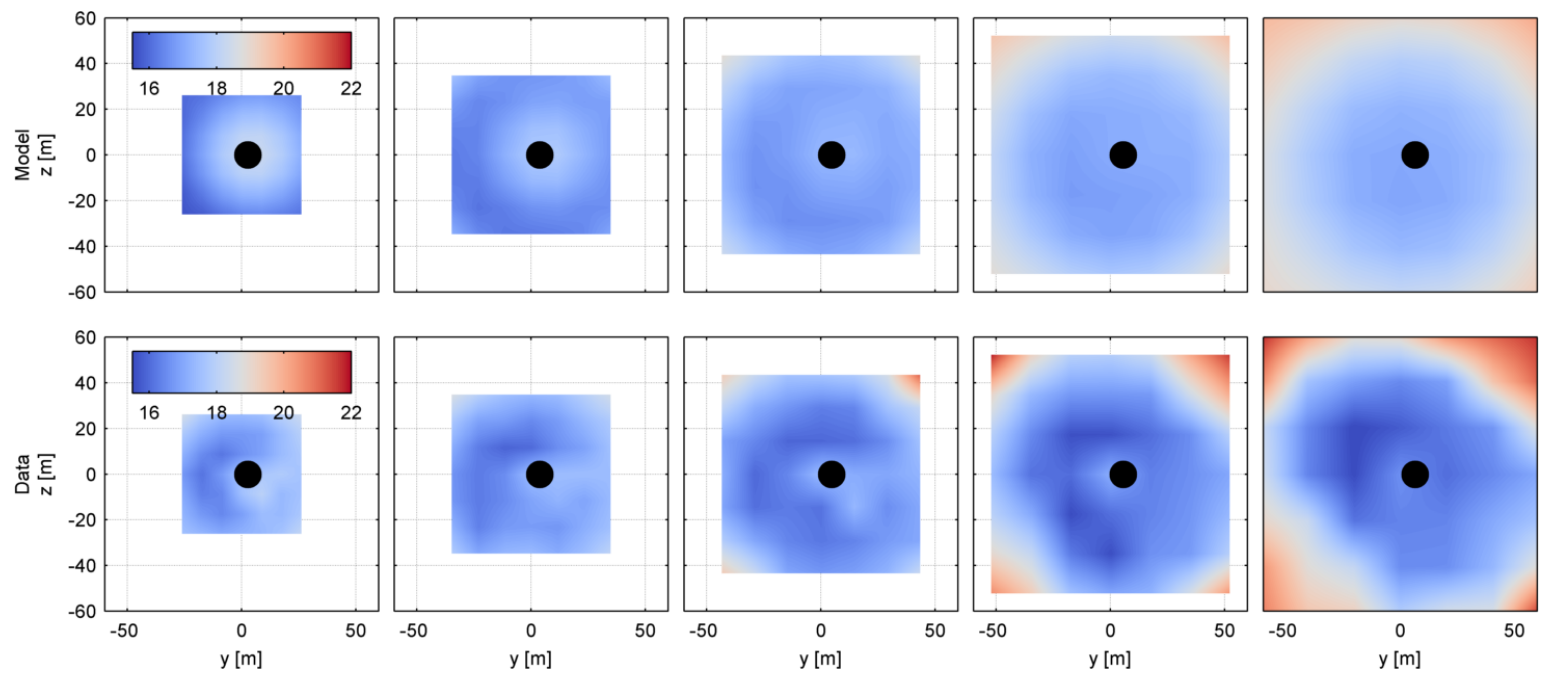

Figure 7. An example estimation step of the wake tracking. The simulated lidar measurements in the first row are compared to the measured lidar data in the second row for five downstream distances from 0.6 to $1.4 \mathrm{D}$ (from left to right, [0.6, 0.8, 1.0, 1.2, 1.4], looking downstream). The estimated wake center is marked with the black dot.

An example initial wake deficit $\Psi_{\text {init }}$ is shown in Fig. 5.

The wake evolves as it moves away from the wind turbine. Energy flows in from the free stream and mixes with the wake. The Navier-Stokes equations describe the flow behavior; however, because of the nonlinearities, using them would be a complex task. Here, an empirical model is used which models the wake recovery. However, in contrast to other wake models, the wake evolution is modeled by a Gaussian shape two-dimensional filter. The two-dimensional filter $\Xi$ depends on the distance $d$ behind the wind turbine

$\Xi\left(d, y_{i}, z_{i}\right)=\exp \left(\frac{y_{i}^{2}+z_{i}^{2}}{2 \sigma_{f}^{2}(d)}\right)$

with

$\sigma_{f}(d)=\frac{d \cdot \epsilon}{2 \sqrt{2 \log (2)}}$

and $y_{i}$ and $z_{i}$ are the grid points in distance $d$. With the parameter $\epsilon$ the dissipation rate can be set.
Thus, for every distance behind the rotor, the wake can be evaluated using the initial wake deficit $\Psi_{\text {init }}$ and the filter (Eq. 7). The wake deficit results from the convolution of the initial wake deficit $\Psi_{\text {init }}$ with the filter $\Xi\left(d, y_{i}, z_{i}\right)$ to

$\Psi\left(d, y_{i}, z_{i}\right)=\Xi\left(d, y_{i}, z_{i}\right) \cdot \Psi_{\text {init }}$.

\subsubsection{Wake deflection model}

The wake deflection caused by a yaw misalignment $\gamma$ is additionally modeled. The relationship is derived in the study by Jiménez et al. (2010) and was successfully used in an optimization of the yaw angles for a simulated wind farm in Gebraad et al. (2014). The angle of the wake with respect to the main wind direction is

$\xi\left(d, c_{\mathrm{T}}, \gamma\right)=\frac{\xi_{\text {init }}\left(c_{\mathrm{T}}, \gamma\right)}{\left(1+\beta \frac{d}{D}\right)^{2}}$, 


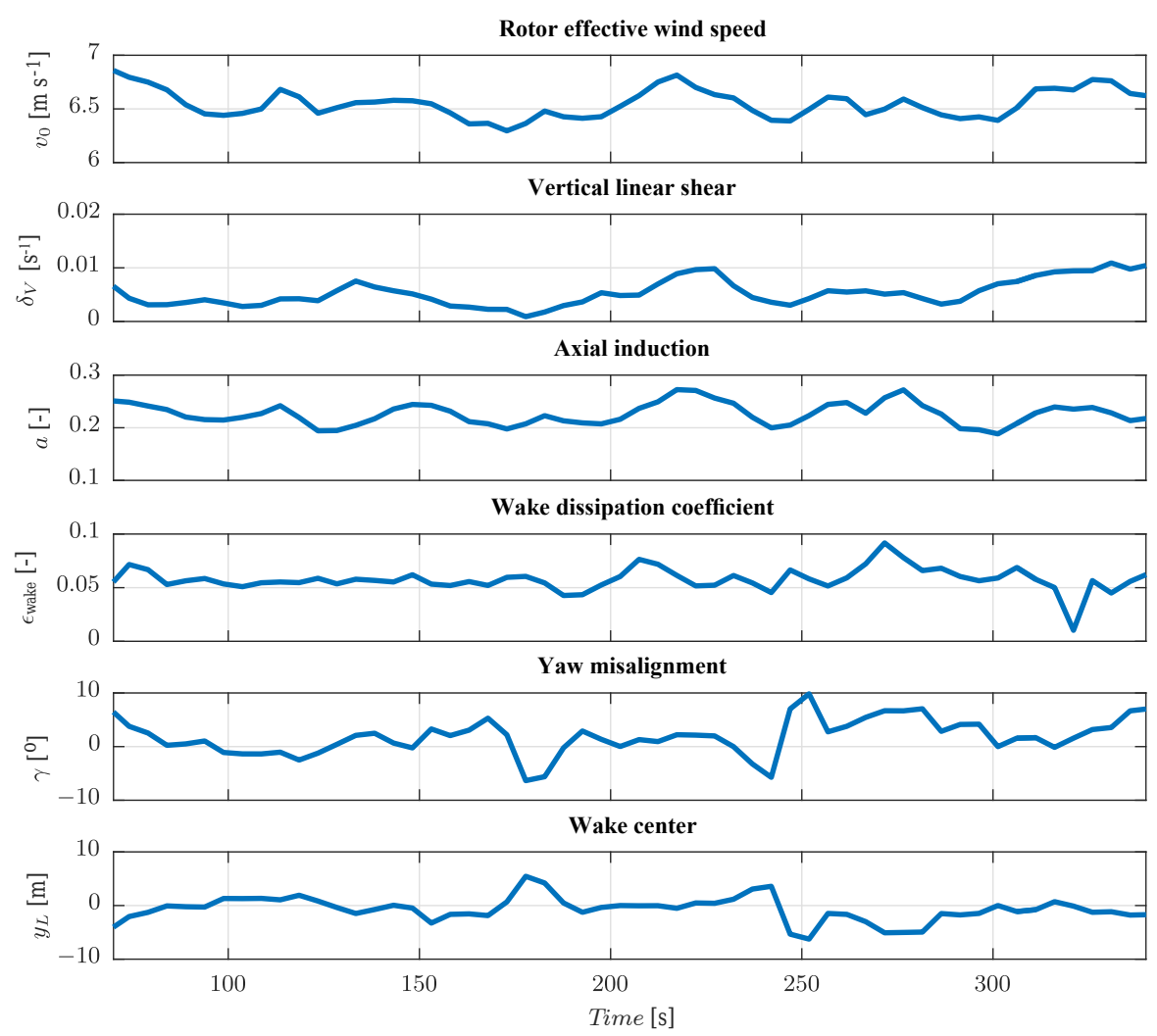

Figure 8. Time results of the wake tracking of a SOWFA simulation. The wind turbine is aligned with the mean wind direction. The lidar scanned in a $7 \times 7$ grid at five distances from 0.6 to $1.4 \mathrm{D}$. The wake center is estimated at the furthest scanning distance $1.4 \mathrm{D}=176.4 \mathrm{~m}$.

with the initial angle of the wake at the rotor

$\xi_{\text {init }}\left(c_{\mathrm{T}}, \gamma\right)=\frac{1}{2} \cos ^{2}(\gamma) \sin (\gamma) c_{\mathrm{T}}$

and model parameter $\beta$, which defines the sensitivity of the wake deflection to yaw and is assumed here to be known in advance. Further, $c_{\mathrm{T}}$ is the thrust coefficient and $D$ is the rotor diameter. The yaw-induced deflection at the downwind position $d$, according to Gebraad et al. (2014), is

$$
\begin{aligned}
\delta_{\text {yaw }}\left(d, c_{\mathrm{T}}, \gamma\right) & =-\xi_{\text {init }}\left(c_{\mathrm{T}}, \gamma\right) \frac{D}{30 \beta}\left[15\left(1-\frac{1}{1+\frac{2 \beta d}{D}}\right)\right. \\
& \left.+\xi_{\text {init }}\left(c_{\mathrm{T}}, \gamma\right)^{2}\left(1-\frac{1}{\left(1+\frac{2 \beta d}{D}\right)^{5}}\right)\right] .
\end{aligned}
$$

The rotation is applied to the wake deficit and yields a $u$ and $v$ component of the wake model:

$$
\left[\begin{array}{c}
\Psi_{u, i} \\
\Psi_{v, i} \\
0
\end{array}\right]_{\mathrm{W}}=\left[\begin{array}{ccc}
\cos \xi & -\sin \xi & 0 \\
\sin \xi & \cos \xi & 0 \\
0 & 0 & 1
\end{array}\right]\left[\begin{array}{c}
\Psi_{i} \\
0 \\
0
\end{array}\right]_{\mathrm{W}}
$$

In Fig. 6 two different wake situations are shown for $\gamma=0^{\circ}$ and $\gamma=25^{\circ}$. The first is a nonyawed case and in the second case the turbine is yawed with $25^{\circ}$. In both cases the underlying wind field has a mean hub-height free-stream wind speed of $v_{0}=16 \mathrm{~m} \mathrm{~s}^{-1}$ and no vertical shear.

\section{The estimation task - model-based wake tracking}

As summarized before, the estimation task tracks the wake using the presented wake model. To perform lidar-based waked tracking, a lidar model is needed. First, the lidar model is presented and then the wake-tracking approach is described. Finally, estimation results of two different cases are presented and discussed.

\subsection{Lidar model}

The lidar measurements can be modeled by a point measurement in the wind field. In the inertial coordinate system this is done by a projection of the wind vector $\left[\begin{array}{lll}u_{i} & v_{i} & w_{i}\end{array}\right]_{\mathrm{I}}^{\mathrm{T}}$ onto the normalized laser vector in the $i$ th point $\left[\begin{array}{lll}x_{i} & y_{i} & z_{i}\end{array}\right]_{\mathrm{I}}^{\mathrm{T}}$ with focus distance $f_{i}=\sqrt{x_{i, \mathrm{I}}^{2}+y_{i, \mathrm{I}}^{2}+z_{i, \mathrm{I}}^{2}}$ by 

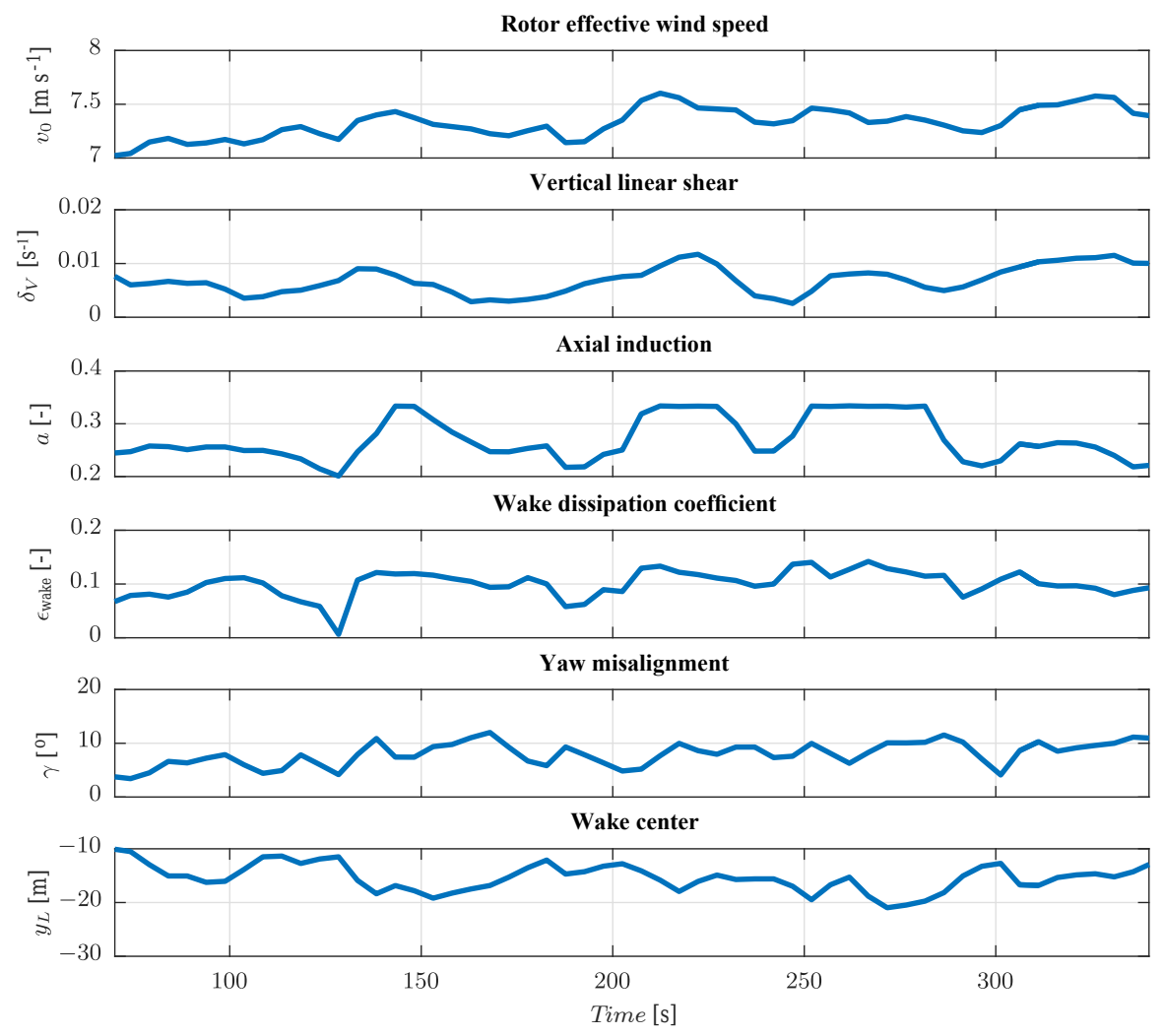

Figure 9. A second time evolution of the different estimated wind field and wake quantities. In this case, the wind turbine is misaligned with $30^{\circ}$ and the wake is deflected. The lidar scanned in a $7 \times 7$ grid at five distances from 0.6 to $1.4 \mathrm{D}$. The wake center is estimated at the furthest scanning distance $1.4 \mathrm{D}=176.4 \mathrm{~m}$.

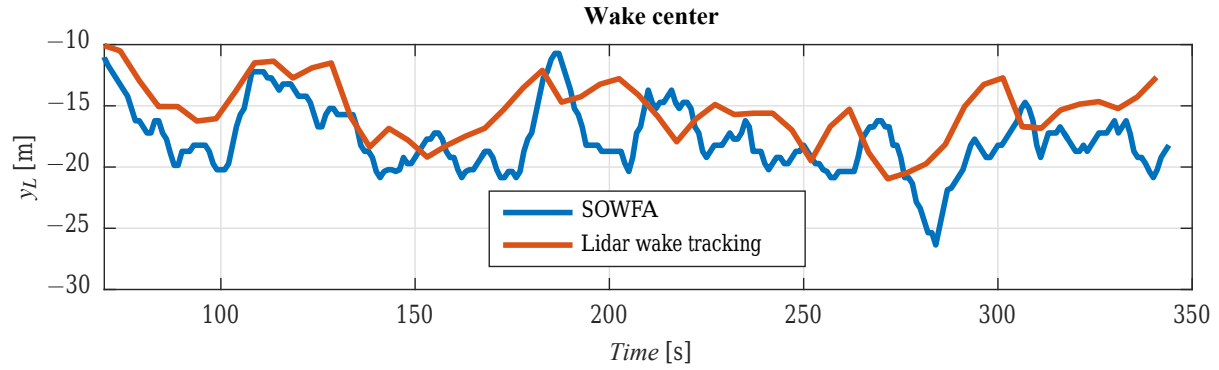

Figure 10. Comparison between the wake center estimation (see Eq. 1) and the lidar-based wake-tracking method.

$v_{\mathrm{los}, i}=\frac{x_{i, \mathrm{I}}}{f_{i}} u_{i, \mathrm{I}}+\frac{y_{i, \mathrm{I}}}{f_{i}} v_{i, \mathrm{I}}+\frac{z_{i, \mathrm{I}}}{f_{i}} w_{i, \mathrm{I}}$.

\subsection{Model-based wake tracking}

As depicted in Fig. 3, the model-based wind field reconstruction method estimates the model parameter by minimizing the error between measured line-of-sight wind speed $v_{\mathrm{los}, \mathrm{m}}$ and simulated line-of-sight wind speed $v_{\text {los, }}$. A nonlinear optimization problem is formed for $n$ measurement points. This yields

$$
\min _{p} f(x)=\min _{p}\left[\begin{array}{c}
\left(v_{\mathrm{los}, \mathrm{m}, 1}-v_{\mathrm{los}, \mathrm{s}, 1}\right)^{2} \\
\vdots \\
\left(v_{\mathrm{los}, \mathrm{m}, n}-v_{\mathrm{los}, \mathrm{s}, n}\right)^{2}
\end{array}\right],
$$

where in $p$ all free model parameters are included. The free model parameters are listed in Table 1. An example of an estimation step of the wake tracking from a measurement campaign at the alpha ventus offshore wind farm is shown in Fig. 7. 
Table 1. The free model parameters for the wind field model which are estimated in the optimizer.

\begin{tabular}{ll|ll}
\hline \multicolumn{2}{c|}{ underlaying wind field } & \multicolumn{1}{c}{ wake model } \\
\hline$v_{0}$ & rotor effective wind speed & $c_{\mathrm{T}}$ & thrust coefficient \\
$\delta_{V}$ & vertical linear shear & $c_{\mathrm{P}}$ & power coefficient \\
& & $\gamma$ & turbine yaw angle \\
& & $\epsilon$ & wake dissipation coefficient \\
\hline
\end{tabular}

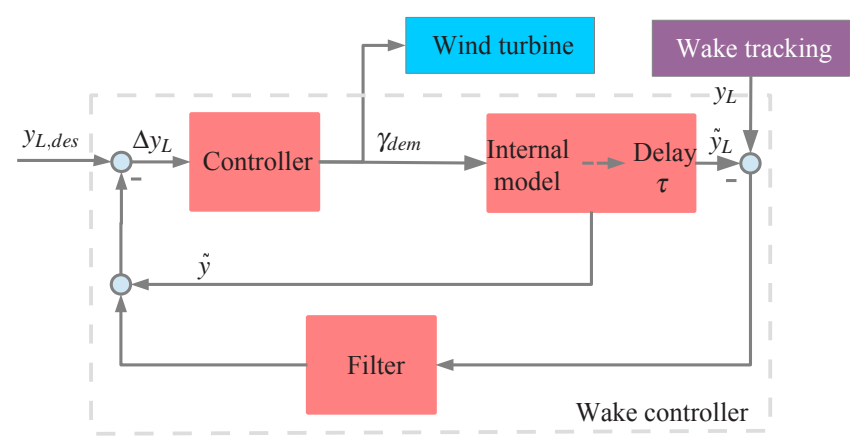

Figure 11. The general structure of the wake steering controller: the controller, a simplified wake model and the wake propagation modeled with a delay, and the filter. The controller uses the difference $\delta y_{L}$, between the predicted output $\tilde{y}$, the measured output $y_{L}$ and the desired output $y_{L \text {,des }}$ to set the demanded yaw angle $\gamma_{\mathrm{dem}}$.

\subsection{Evaluation and discussion}

Figure 7 confirms the applicability of the method with lidar measurement data. In the following, SOWFA (Churchfield and Lee, 2012) is used as a simulation tool. Flow snapshots of simulations of a single wind turbine are stored. The flow field is then scanned with a lidar simulator. The lidar scans with a $7 \times 7$ grid at five distances from 0.6 to $1.4 D$ (with $D=$ $126 \mathrm{~m}$ ). Two different cases are analyzed. In the first case, the turbine rotor is perpendicular to the wind direction $\left(\gamma=0^{\circ}\right)$ and these results are shown in Fig. 8. In the second case, the yaw misalignment is $30^{\circ}$ so that the wake is deflected. The results of the wake tracking are shown in Fig. 9. In both figures the wake center is estimated at the furthest scanning distance of $1.4 D=176.4 \mathrm{~m}$. In both cases the method shows the ability to estimate the wake parameter and tracking its center.

As mentioned before, the wake center position needs to be calculated using a specific definition and there is no direct measurable representation of it. In Fig. 10 the lidar-based wake tracking is compared to the wake center estimation using the definition of Eq. (1) without any filtering.

\section{The control task}

The following closed-loop controller was first presented in Raach et al. (2016) and is recapped here. See also Raach et al. (2017), in which a $\mathcal{H}_{\infty}$ controller design for closed- loop wake redirection with defined performance margins is used. As mentioned above, the reaction of the wake to a yaw action can only be measured with a time delay. To control a delayed system, the Smith predictor approach, which is based on internal model control, has been derived and used in many applications.

The presented controller follows the idea of internal model control in which the difference between the actual system output and a predicted output is used within the controller to regulate the system. Therefore, a model is necessary for describing the wake effects in a simplified but sufficient way. It consists of a proportional-integral (PI) controller. Further, an internal model is used which approximates the real system behavior. The wake propagation which exists because the wake flow has to evolve until it reaches the measurement location of the lidar system is modeled using a time delay. The delay time $\tau$ varies with respect to the mean wind speed. Finally, a filter is needed to cancel out controller actions which can not be observed because of the time difference between control action and measurement location. Figure 11 shows the general concept of the controller.

\subsection{Internal wake model of the controller}

As depicted in Fig. 11, the wake controller needs an internal model to predict the reaction of the wake to the demanded yaw angle. The internal wake model includes the yaw actuator and the gain between the yaw and the wake deflection. For the wake model the assumptions of a constant $c_{\mathrm{T}}$ is made. Altogether, this yields an internal controller model $\widetilde{\Psi}$ of the reality $\Psi$ :

$$
\widetilde{\Psi}:\left\{\begin{array}{l}
\ddot{\gamma}+2 d \omega \dot{\gamma}+\omega^{2} \gamma=\omega^{2} \gamma_{\mathrm{dem}} \\
\tilde{y}=\delta_{\text {yaw }}\left(d_{\text {Lidar }}, c_{\mathrm{T}, \mathrm{const}}, \gamma\right)
\end{array} \mid,\right.
$$

with $\gamma_{\text {dem }}$ being the demanded yaw angle and $d_{\text {Lidar }}$ the distance to the measurement location. Equation (16), line 1 is the yaw actuator dynamic and Eq. (16), line 2 the wake deflection model.

There is a time delay because the wake first needs to evolve to the measurement location:

$\tilde{y}_{L}(t)=\tilde{y}(t-\tau)$.

For the controller design, the time delay is approximated using the Padé approximation of time delays; see Skogestad and Postlethwaite (2005). 

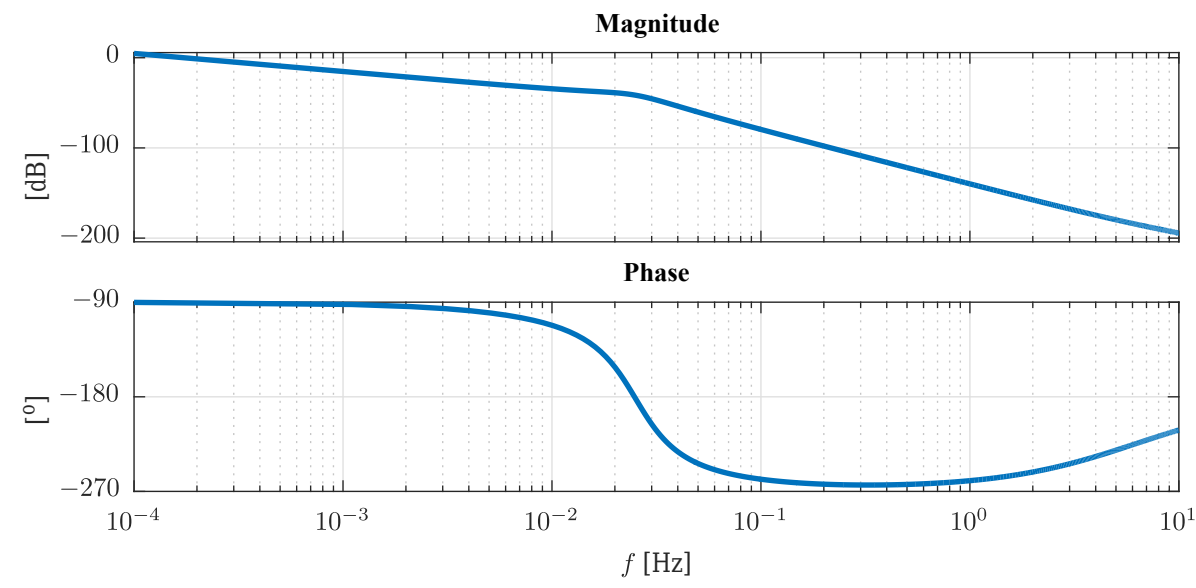

Figure 12. Bode analysis of the designed controller $K$.
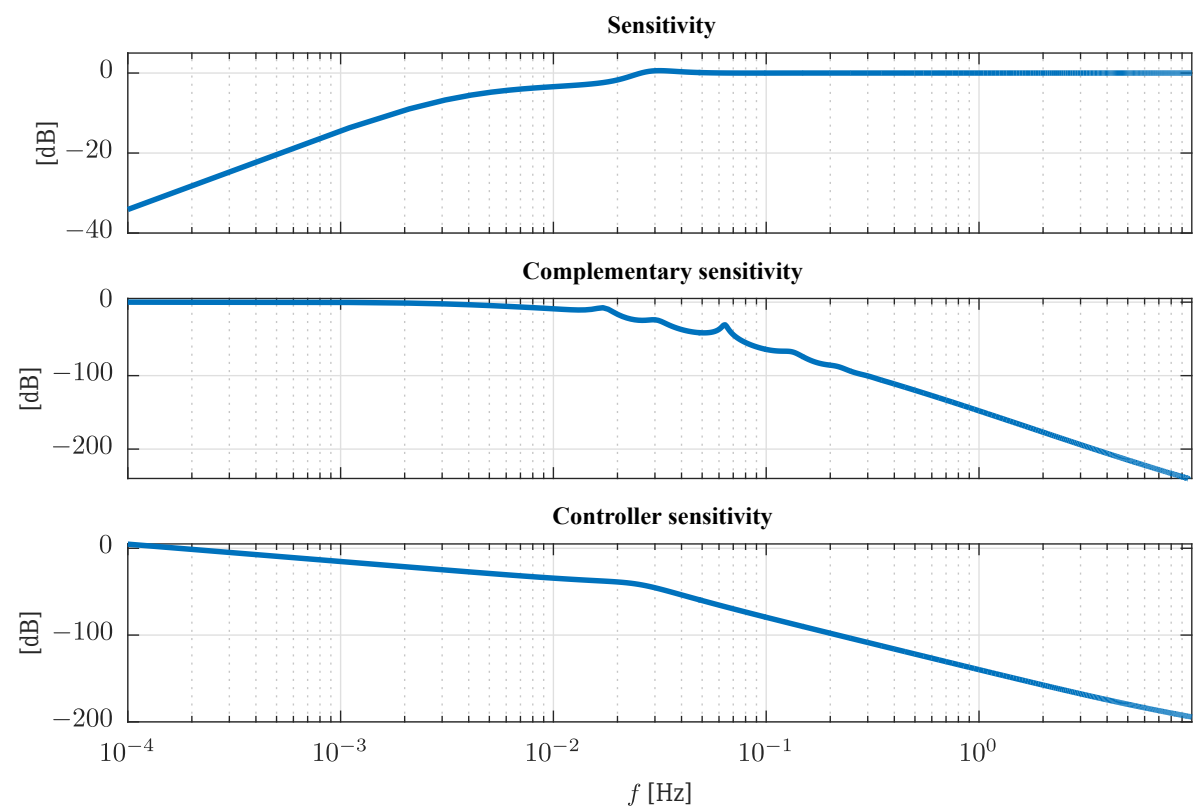

Figure 13. Sensitivity $S$, complementary sensitivity $T$, and controller sensitivity $U$ analysis of the closed-loop system.

\subsection{Controller design}

The primal goal of the wake controller is to steer the wake center to a desired point in a defined distance by yawing the wind turbine. As mentioned, this is done using a Smith predictor, which uses an internal model to predict the output reaction. Then the predicted wake center position and the filtered error between predicted and measured wake center position is fed back to the controller.

\subsubsection{Controller}

A standard PI controller is used. It is designed such that the closed-loop performance with the internal model (Eq. 16) meets a phase margin of $60^{\circ}$ and a closed-loop bandwidth of $\omega_{\mathrm{CL}}=\frac{1}{2 \tau}$. This yields a controller of the form

$u=K_{\mathrm{p}}\left(\Delta y_{L}+\frac{1}{T_{i}} \int \Delta y_{L} \mathrm{~d} t\right)$,

with the proportional gain $K_{\mathrm{p}}$, the time constant $T_{i}$, and the error between desired and actual wake center position $\Delta y_{L}$.

\subsubsection{Filter}

The wake propagation and the caused delay precludes direct measurement of a yaw change, and because of that one has to filter the measured feedback to prevent nonobservable yaw actions. Since the delay $\tau$ is time varying and depends on the mean wind speed, the filter has to be adaptable. Therefore, 
the cutoff frequency of the butterworth low-pass filter is set to $\omega_{\text {filter }}=\frac{\pi}{8 \tau}$.

\subsection{Evaluation and discussion}

In the following the wake controller is analyzed. Further, the sensitivity, the complementary sensitivity, and the controller sensitivity of the closed-loop system are assessed. Considering Skogestad and Postlethwaite (2005), the sensitivity gives the closed-loop transfer function from the output disturbance to the output while the complementary sensitivity is the closed-loop transfer function from the reference to the output. The controller sensitivity gives the closed-loop transfer function from the output disturbance to the controller. For more details and a description on controller design and analysis, we refer to Skogestad and Postlethwaite (2005).

\subsubsection{Controller analysis}

In the following, the transfer function of the wake controller is assessed. As shown in Fig. 11 the wake controller consists of the internal controller $C$, an internal model $\widetilde{\Psi}$, the time delay approximation $W$, and the filter $F$. Having merged all parts the wake controller $K$ is

$$
K=\frac{F}{(1+C \Psi(1-F W))} .
$$

Figure 12 shows the bode analysis of the wake controller $K$. The controller shows integration behavior, starting with $-90^{\circ}$ phase.

\subsubsection{Closed-loop analysis}

To perform closed-loop analysis the internal controller model $\widetilde{\Psi}$ is transformed to Laplacian space, yielding the plant $G$. Then, the sensitivity $S$, the complementary sensitivity $T$, and the controller sensitivity $U$ that are

$$
\begin{aligned}
S & =\frac{1}{1+G K} \\
T & =\frac{G K}{1+G K} \\
U & =\frac{K}{1+G K},
\end{aligned}
$$

with the controller $K$ are assessed and shown in Fig. 13. The sensitivity shows a disturbance attenuation up to the controller bandwidth $\omega_{\mathrm{CL}}=0.02 \mathrm{~Hz}$. Further, the controller sensitivity has low gain for high frequencies. This means the controller does not react to high-frequency disturbances.

\section{Conclusions}

This paper first introduces a method which uses lidar measurements to estimate wind field parameters and enables tracking of the wake center position. Second, a controller is presented which uses this information to redirect the wake to a desired position. In two different cases using simulated lidar measurements of SOWFA simulations, the wake tracking shows promising results in estimating the wake center. The difficulty in wake center position definition is elaborated. A definition is used and the wake-tracking results are compared to it. The challenges of a lidar-based wake redirection control problem are discussed and an appropriate controller is designed to meet the desired requirements. This leads to the next step, towards closed-loop wake redirection in a highfidelity simulation tool.

The presented framework of lidar-based closed-loop wake steering offers new possibilities for wind farm control. In the future, a balance between measuring the near wake, which will result in a higher controller bandwidth, and the far wake, which will give more reliable information on the wake direction, needs to be found. In a next step, it will be implemented and tested in a high-fidelity simulation tool and tested in real time. For the control problem, robust controllers will be investigated. Dynamic estimation techniques as well as other wake estimation models will be used for comparing the ability of tracking the wake and finding the most suitable approach for this task.

Data availability. The SOWFA data used in this work have been provided by the National Reneawable Energy Laboratory (NREL) and is publicly available at http://wind.nrel.gov/public/ssc/.

Competing interests. The authors declare that they have no conflict of interest.

Acknowledgements. The authors would like to acknowledge the CL-Windcon project which has received funding from the European Union's Horizon 2020 research and innovation programme under grant agreement No 727477. Further, we would like to thank Pieter Gebraad and Paul Fleming for fruitful discussions and providing the SOWFA data set.

Edited by: C. L. Bottasso

Reviewed by: two anonymous referees

\section{References}

Boersma, S., Doekemeijer, B., Gebraad, P., Fleming, P., Annoni, J., Scholbrock, A., Frederik, J., and van Wingerden, J.-W.: A Tutorial on Control-Oriented Modeling and Control of Wind Farms, in: Proceedings of the American Control Conference (ACC), Seattle, USA, 2017.

Borisade, F., Luhmann, B., Raach, S., and Cheng, P. W.: Shadow Effects in an Offshore Wind Farm - Potential of Vortex Methods for Wake Modelling, in: Proceedings of the German Wind Energy Conference DEWEK, Bremen, Germany, 2015. 
Churchfield, M. and Lee, S.: NWTC design codes-SOWFA, available at: http://wind.nrel.gov/designcodes/simulators/SOWFA (last access: 15 May 2017), 2012.

Doubrawa, P., Barthelmie, R. J., Wang, H., and Churchfield, M. J.: A stochastic wind turbine wake model based on new metrics for wake characterization, Wind Energy, 20, 449-463, doi:10.1002/we.2015, 2017.

Fleming, P., Gebraad, P. M., Lee, S., Wingerden, J.-W., Johnson, K., Churchfield, M., Michalakes, J., Spalart, P., and Moriarty, P.: Simulation comparison of wake mitigation control strategies for a two-turbine case, Wind Energy, 18, 2135-2143, 2014a.

Fleming, P. A., Gebraad, P. M., Lee, S., van Wingerden, J.-W., Johnson, K., Churchfield, M., Michalakes, J., Spalart, P., and Moriarty, P.: Evaluating techniques for redirecting turbine wakes using SOWFA, Renew. Energ., 70, 211-218, 2014b.

Gebraad, P. M. O., Teeuwisse, F. W., van Wingerden, J., Fleming, P., Ruben, S. D., Marden, J. R., and Pao, L. Y.: Wind plant power optimization through yaw control using a parametric model for wake effects - a CFD simulation study, Wind Energy, 19, 95114, doi:10.1002/we.1822, 2014.

Howland, M. F., Bossuyt, J., Martinez-Tossas, L. A., Meyers, J., and Meneveau, C.: Wake Structure of Wind Turbines in Yaw under Uniform Inflow Conditions, available at: http://arxiv.org/abs/ 1603.06632 (last access: 15 May 2017), 2016.

Jiménez, Á., Crespo, A., and Migoya, E.: Application of a LES technique to characterize the wake deflection of a wind turbine in yaw, Wind Energy, 13, 559-572, 2010.

Lundquist, J. K., Churchfield, M. J., Lee, S., and Clifton, A.: Quantifying error of lidar and sodar Doppler beam swinging measurements of wind turbine wakes using computational fluid dynamics, Atmos. Meas. Tech., 8, 907-920, doi:10.5194/amt-8-907$2015,2015$.
Raach, S., Schlipf, D., Haizmann, F., and Cheng, P. W.: Three Dimensional Dynamic Model Based Wind Field Reconstruction from LiDAR Data, in: Journal of Physics: Conference Series: The Science of Making Torque From Wind, Vol. 524, Copenhagen, Denmark, 2014.

Raach, S., Schlipf, D., Borisade, F., and Cheng, P. W.: Wake redirecting using feedback control to improve the power output of wind farms, in: Proceedings of the American Control Conference (ACC), Boston, USA, 2016.

Raach, S., van Wingerden, J.-W., Boersma, S., Schlipf, D., and Cheng, P. W.: Hinf controller design for closed-loop wake redirection, in: Proceedings of the American Control Conference (ACC), Seattle, USA, 2017.

Schlipf, D., Rettenmeier, A., Haizmann, F., Hofsäß, M., Courtney, M., and Cheng, P. W.: Model Based Wind Vector Field Reconstruction from Lidar Data, in: Proceedings of the German Wind Energy Conference DEWEK, Bremen, Germany, 2012.

Skogestad, S. and Postlethwaite, I.: Multivariable Feedback Control: Analysis and Design, John Wiley \& Sons, New York, USA, 2005.

Vollmer, L., Steinfeld, G., Heinemann, D., and Kühn, M.: Estimating the wake deflection downstream of a wind turbine in different atmospheric stabilities: an LES study, Wind Energ. Sci., 1, 129 141, doi:10.5194/wes-1-129-2016, 2016. 